\title{
Unusual thrombus formation in the aorta after apicoaortic conduit for severe aortic stenosis
}

Koji Takeda, MD, Goro Matsumiya, MD, Hiroshi Takano, MD, Masahiro Koh, MD, Keiji Iwata, MD, and

Yoshiki Sawa, MD, Osaka, Japan

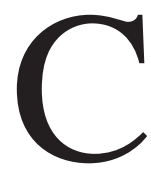

onventional valve replacement for aortic valve stenosis has significant operative risks in patients with dense, circumferential calcification of the ascending aorta. The apicoaortic conduit (AAC) is one of the alternative methods to eliminate the aortic manipulation. Several reports have demonstrated that a newly constructed double-outlet left ventricle maintains normally distributed blood flow throughout the systemic circulation without any deleterious physiologic effects. ${ }^{1,2}$ However, we encountered the case of a patient who had unanticipated thrombus formation in the aortic arch that might have resulted from fractionation of the cardiac output and stagnation of the blood flow after construction of the AAC.

\section{Clinical Summary}

A 72-year-old woman with a history of diabetes mellitus and chronic renal insufficiency requiring hemodialysis experienced progressive dyspnea on exertion. Echocardiography demonstrated severe aortic stenosis caused by a calcified valve. Cardiac catheterization demonstrated a pressure gradient of $58 \mathrm{~mm} \mathrm{Hg}$ across the aortic valve, a cardiac index of $3.5 \mathrm{~L} \cdot \mathrm{min}^{-1} \cdot \mathrm{m}^{-2}$, and $90 \%$ stenosis of the proximal right coronary artery. Computed tomography showed severe circumferential aortic calcification of the whole aorta, including the aortic root.

To preclude the aortic valve procedure through a median sternotomy, we inserted the AAC through the left thoracotomy. The thoracic cavity was entered through the sixth intercostal space, and cardiopulmonary bypass was initiated with distal descending aortic cannulation and right femoral venous drainage. The patient was kept normothermic, and a 20-mm Hemashield graft (Meadox, Hemashield; Boston Scientific, Boston, Mass) was sewn to the descending aorta during side clamping. Continuous infusion of $\beta$-blocker was started, and the heart rate was decreased to around 30 beats/min. In a head-down position, the left ventricular apex was enucleated with a $20-\mathrm{mm}$ coring knife. The valved conduit incorporating a 23-mm stentless valve (Edwards Prima Plus; Stentless Bioprosthesis, Inc, Irvine, Calif) and a 20-mm Hemashield graft was anastomosed with the apical hole. The

From the Division of Cardiovascular Surgery, Department of Surgery, Osaka University Graduate School of Medicine, Osaka, Japan,

Received for publication Feb 10, 2006; accepted for publication Feb 22, 2006.

Address for reprints: Goro Matsumiya, MD, Division of Cardiovascular Surgery, Department of Surgery, Osaka University Graduate School of Medicine, 2-2 Yamadaoka, Suita, Osaka 565-0871, Japan (E-mail: matsumg@surg1.med.osaka-u.ac.jp).

J Thorac Cardiovasc Surg 2006;132:155-6

$0022-5223 / \$ 32.00$

Copyright $(2006$ by The American Association for Thoracic Surgery doi:10.1016/j.jtcvs.2006.02.044
2 parts of the conduit were then anastomosed. A saphenous vein graft placed from the coronary orifice of the stentless valve was grafted to the right coronary artery concomitantly.

The postoperative course was uncomplicated, and the patient had improvement of symptoms. Computed tomography at 1 month after the operation demonstrated unobstructed conduit (Figure 1) and unexpected thrombus formation, possibly caused by stagnation of blood flow in the aortic arch (Figure 2). Administration of sodium warfarin was initiated immediately, and no thromboembolic events have been observed.

\section{Discussion}

Recent modifications of the technique and innovation of the technology has made it more feasible to accomplish the AAC for various complex lesions of the left ventricular outflow tract not easily amenable to other techniques. ${ }^{3,4}$ In our case creation of an AAC combined with coronary revascularization was successfully performed with a pharmacologically controlled beating heart, which is a new and lessinvasive procedure even with the support of cardiopulmonary bypass. Furthermore, the use of a stentless bioprosthesis enabled us to obtain a larger valve orifice and to avoid anticoagulation. The patient was freed from heart failure symptoms, and postoperative computed to-

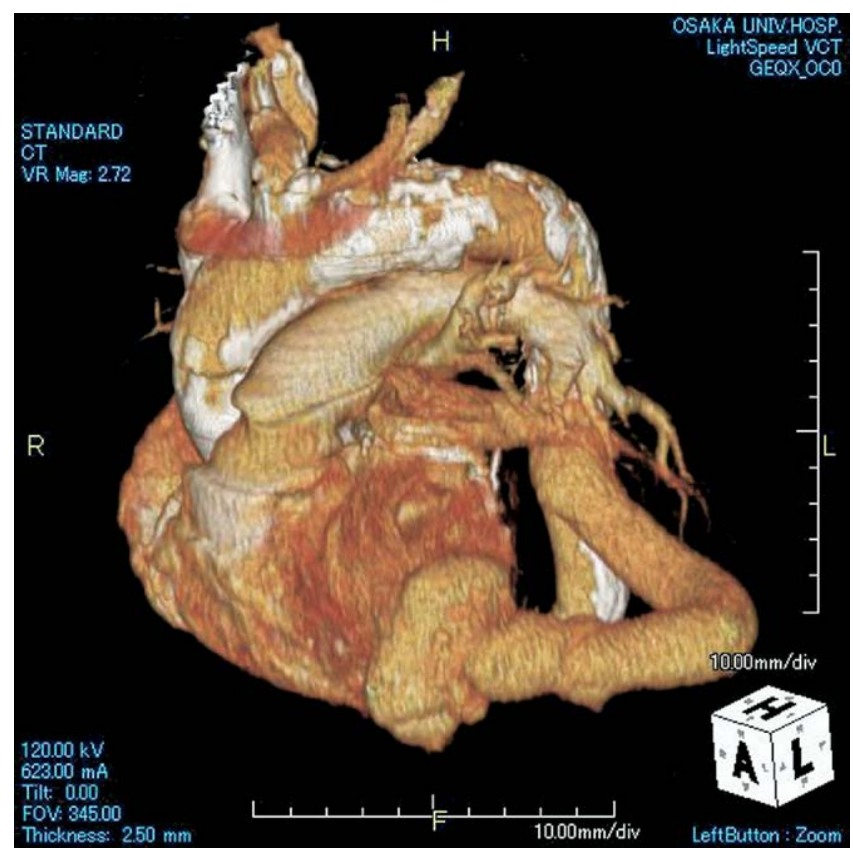

Figure 1. Postoperative computed tomography after apicoaortic conduit insertion demonstrated an unobstructed conduit. 

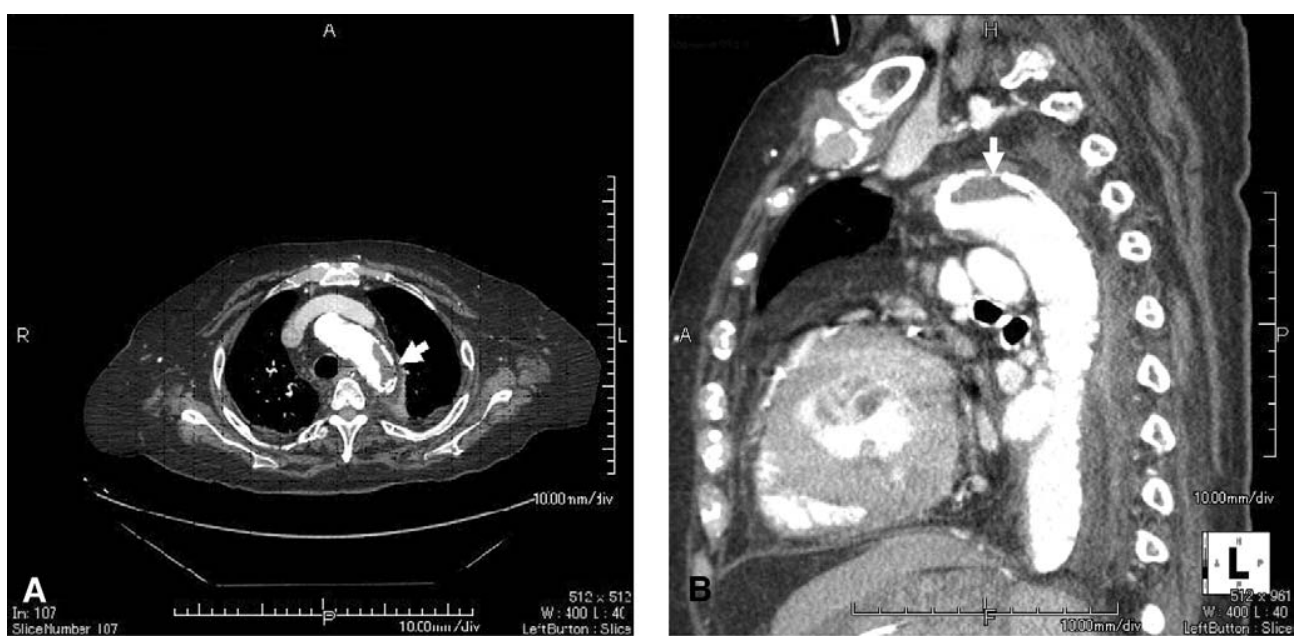

Figure 2. Axial (A) and sagittal (B) view of the thrombus formation (arrow) in the aortic arch after apicoaortic conduit insertion.

mography demonstrated unobstructed conduit without any sign of intraconduit thrombus.

There have been concerns about the fractionation of the cardiac output after construction of the AAC. Few previous studies have demonstrated that blood flow is distributed normally throughout the systemic and coronary circulation, and no deleterious effects have resulted from fractionation of the cardiac output into the antegrade (native outflow) and retrograde (conduit) portions. ${ }^{1,2}$ However, recent experiences with a left ventricular assist system, which bypasses the left ventricle and the descending aorta, have shown the stagnation of blood flow in the ascending aorta and the resultant massive myocardial infarction from coronary artery occlusion in a few patients. ${ }^{5}$ The same scenario could take place in the patients receiving an AAC. The flow through the conduit depends on the degree of the native left ventricular outflow tract obstruction in relation to the conduit size and its inherent impedance. ${ }^{1}$ In the present case preoperative cardiac output was relatively preserved (cardiac index of $3.5 \mathrm{~L} \cdot \mathrm{min}^{-1}$. $\mathrm{m}^{-2}$ ). Therefore the antegrade flow after construction of the AAC might have been maintained in relation to the retrograde flow, and that has led to stagnation of the bloodstream and the subsequentf thrombus formation in the aortic arch. Although the patient had no embolic events, further predominance of retrograde flow might have caused a cerebral infarction. In this point of view, close observation of the thrombus formation in the aorta and postoperative anticoagulation therapy might be warranted after the AAC, even in patients who received biologic valves.

\section{References}

1. Norman JC, Nihill MR, Cooly DA. Valved apico-aortic composite conduits for left ventricular outflow tract obstructions. A 4 year experience with 27 patients. Am J Cardiol. 1980;45:1265-71.

2. Nihill MR, Cooley DA, Norman JC, Hallman GL, McNamara DG Hemodynamic observations in patients with left ventricle to aorta conduit. Am J Cardiol. 1980;45:573-82.

3. Cooly DA, Lopez RM, Absi TS. Apicoaortic conduit for left ventricular outflow tract obstruction: revised. Ann Thorac Surg. 2000;69:1511-4.

4. Vassiliades TA Jr. Off-pump apicoaortic conduit insertion for high-risk patients with aortic stenosis. Eur J Cardiothorac Surg. 2003;23:156-8.

5. Frazier OH, Myers TJ, Westaby S, Gregoric ID. Clinical experience with an implantable, intracardiac, continuous flow circulatory support device: physiologic implications and their relationship to patient selection. Ann Thorac Surg. 2004;77:133-42. 